\title{
Oscillatory Brain State Predicts Variability in Working Memory
}

\author{
Nicholas E. Myers, Mark G. Stokes, Lena Walther, and Anna C. Nobre \\ Department of Experimental Psychology and, Oxford Centre for Human Brain Activity, University of Oxford, Oxford OX1 3UD, United Kingdom
}

Our capacity to remember and manipulate objects in working memory (WM) is severely limited. However, this capacity limitation is unlikely to be fixed because behavioral models indicate variability from trial to trial. We investigated whether fluctuations in neural excitability at stimulus encoding, as indexed by low-frequency oscillations (in the alpha band, $8-14 \mathrm{~Hz}$ ), contribute to this variability. Specifically, we hypothesized that the spontaneous state of alpha band activity would correlate with trial-by-trial fluctuations in visual WM. Electroencephalography recorded from human observers during a visual WM task revealed that the prestimulus desynchronization of alpha oscillations predicts the accuracy of memory recall on a trial-by-trial basis. A model-based analysis indicated that this effect arises from a modulation in the precision of memorized items, but not the likelihood of remembering them (the recall rate). The phase of posterior alpha oscillations preceding the memorized item also predicted memory accuracy. Based on correlations between prestimulus alpha levels and stimulus-related visual evoked responses, we speculate that the prestimulus state of the visual system prefigures a cascade of state-dependent processes, ultimately affecting WM-guided behavior. Overall, our results indicate that spontaneous changes in cortical excitability can have profound consequences for higher visual cognition.

Key words: alpha oscillations; cognitive variability; cortical excitability; precision; working memory

\section{Introduction}

Visual working memory (WM) allows us to maintain and manipulate visual information over brief periods of time and is critical for guiding ongoing behavior. However, the storage capacity of WM is extremely limited (Cowan, 2000), either in the quality (Bays et al., 2009; Keshvari et al., 2013) or quantity (i.e., 3-4 items: Zhang and Luck, 2008; Luck and Vogel, 2013) of information that can be maintained. Such capacity limits have profound implications. Individual differences in WM performance strongly predict higher cognitive functions such as IQ (Engle et al., 1999; Fukuda et al., 2010) and real-world performance (Gathercole et al., 2003).

Interestingly, WM capacity may not be a static trait even within an individual. Recent studies measuring the fidelity of representations stored in WM reveal significant trial-by-trial variability within observers, both in the number of encoded items (Sims et al., 2012)

Received Nov. 7, 2013; revised Jan. 28, 2014; accepted Feb. 24, 2014.

Author contributions: N.E.M., M.G.S., L.W., and A.C.N. designed research; N.E.M. and L.W. performed research; N.E.M. analyzed data; N.E.M., M.G.S., L.W., and A.C.N. wrote the paper.

This work was supported by the Wellcome Trust (N.E.M., L.W., and A.C.N.), the Medical Research Council (M.G.S), and the National Institute for Health Research Oxford Biomedical Research Centre Programme (A.C.N. and M.G.S.) based at the $0 x$ ford University Hospitals Trust, 0 xford University. The views expressed are those of the author(s) and not necessarily those of the NHS, the NIHR, or the Department of Health. We thank Nils Kolling, George Wallis, and Gustavo Rohenkohl for helpful discussions.

The authors declare no competing financial interests.

This article is freely available online through the J Neurosci Author Open Choice option.

Correspondence should be addressed to Nicholas E. Myers, Department of Experimental Psychology and Oxford Centre for Human Brain Activity, University of Oxford, 9 South Parks Road, Oxford OX1 3UD, UK. E-mail: nicholas.myers@psy.ox.ac.uk.

DOI:10.1523/JNEUROSCI.4741-13.2014

Copyright $\odot 2014$ Myers et al.

This is an Open Access article distributed under the terms of the Creative Commons Attribution License (http://creativecommons.org/licenses/by/3.0), which permits unrestricted use, distribution and reproduction in any medium provided that the original work is properly attributed. and in how precisely they are represented (Fougnie et al., 2012; van den Berg et al., 2012; Orhan and Jacobs, 2013). Previous modeling studies have been limited to the behavioral phenomenon of variability in visual WM and only speculated on its neural causes.

We hypothesized that variation in the state of excitability of sensory neural systems could contribute to the variability of encoding into WM. Slow neural oscillations have been proposed to reflect dynamic changes in the state of cortical excitation (Klimesch et al., 2007; Wyart and Sergent, 2009; Jensen and Mazaheri, 2010). In particular, alpha-band oscillations (between 8 and $14 \mathrm{~Hz}$ ) correlate with visual excitability, such that firing rates in response to visual stimulation are reduced by neural synchronization in the alpha band (Haegens et al., 2011; Spaak et al., 2012) and at specific phases of the alpha cycle (Bollimunta et al., 2011; Saalmann et al., 2012). Correspondingly, the top-down reduction of alpha band power using attention-guiding cues has been shown to improve the detection of liminally presented stimuli (van Dijk et al., 2008; Mathewson et al., 2011) and to speed reaction times in spatial cueing tasks (Gould et al., 2011). Studies of visual perception have also shown that, in the absence of task-relevant cues, the detectability of stimuli covaries with spontaneous fluctuations in the prestimulus power (Hanslmayr et al., 2007; Romei et al., 2008; VanRullen and Macdonald, 2012) and phase (Busch and VanRullen, 2010; Chakravarthi and VanRullen, 2012) of alpha oscillations. Whether such spontaneous changes in the state of visual excitability can have longer-lasting consequences for WM has not been established.

Here, we tested this idea by investigating whether prestimulus alpha oscillations measured with electroencephalography (EEG) influence the encoding of items into WM. Our task (Fig. 1A) was optimized for detecting oscillatory states that relate to individual items by presenting stimuli in sequence (Gorgoraptis et al., 2011). 
Further, we used a free-recall paradigm that allowed us to measure subtle trial-bytrial fluctuations in the precision of memory (Zhang and Luck, 2008). This experimental approach allowed us to test whether any influences of alpha might independently modulate the precision of a WM representation or its likelihood of being remembered.

\section{Materials and Methods}

Task design. We tested 17 healthy right-handed volunteers ( 9 females, mean age 22.9 years, range 20-30 years) during a sequential visual WM task (Fig. 1A). Each participant finished two sessions, conducted on separate days, consisting of 500 trials each. On each trial, participants saw a stream of 3-4 oriented bars (each displayed for $150 \mathrm{~ms}$ ) appearing at random locations on an imaginary circle ( $6^{\circ}$ of visual angle from a fixation dot, which subtended $0.30^{\circ}$ ), with a stimulus-onset asynchrony uniformly jittered between 950 and $1200 \mathrm{~ms}$. After a memory delay following the last shape (950$1200 \mathrm{~ms}$ for 4 -item trials, $1900-2400 \mathrm{~ms}$ for 3 -item trials), a probe appeared at a previous stimulus location. The probe consisted of a black circle with a diameter of the same length as the bars. After participants started moving the mouse, two small tracking balls appeared $180^{\circ}$ apart at a random location on the circle. Participants rotated the tracking balls to the remembered orientation at the probed location.

Stimuli. Stimuli were oriented bars with a length of $1.40^{\circ}$ and a width of $0.32^{\circ}$. To emphasize the point of rotation of the stimuli, the center of the bar contained a circular disc with a diameter of $0.68^{\circ}$. Some trials $(30 \%)$ contained a distractor stimulus at the end of the sequence. Distractors were never probed and participants were encouraged to ignore them. Target shapes were distinguished from distractors by their color (isoluminant blue and green, counterbalanced across sessions).

Behavioral analysis. For each trial, we calculated the deviation (in degrees) of the response from the actual orientation of the probed stimulus, generating a response distribution across trials. Initially, we calculated memory precision as the inverse of the circular SD of the response distribution (corrected for the precision expected from randomly distributed responses; Bays et al., 2009), separately for each condition (i.e., the 10 combinations of memory load ( 3 or 4 items), distractor presence, and position of the probed item in the sequence (first, second, third, or fourth). To get a more revealing measure of memory, we also treated observers' responses as drawn from a mixture of two distributions as follows:

$$
p(x \mid \theta)=\frac{P_{M E M}}{2 \pi I_{0}(\kappa)} e^{\kappa \cos (x-\theta)}+\frac{1-P_{M E M}}{2 \pi},
$$

where $x$ is the response angle, $\theta$ is the presented angle, and $I_{0}$ is the modified Bessel function (of order 0 ). The two variable parameters, $P_{\mathrm{MEM}}$ and $\kappa$, define the two distributions, respectively: the probability of recalling an item $\left(P_{\mathrm{MEM}}\right)$ and the concentration parameter, or precision,
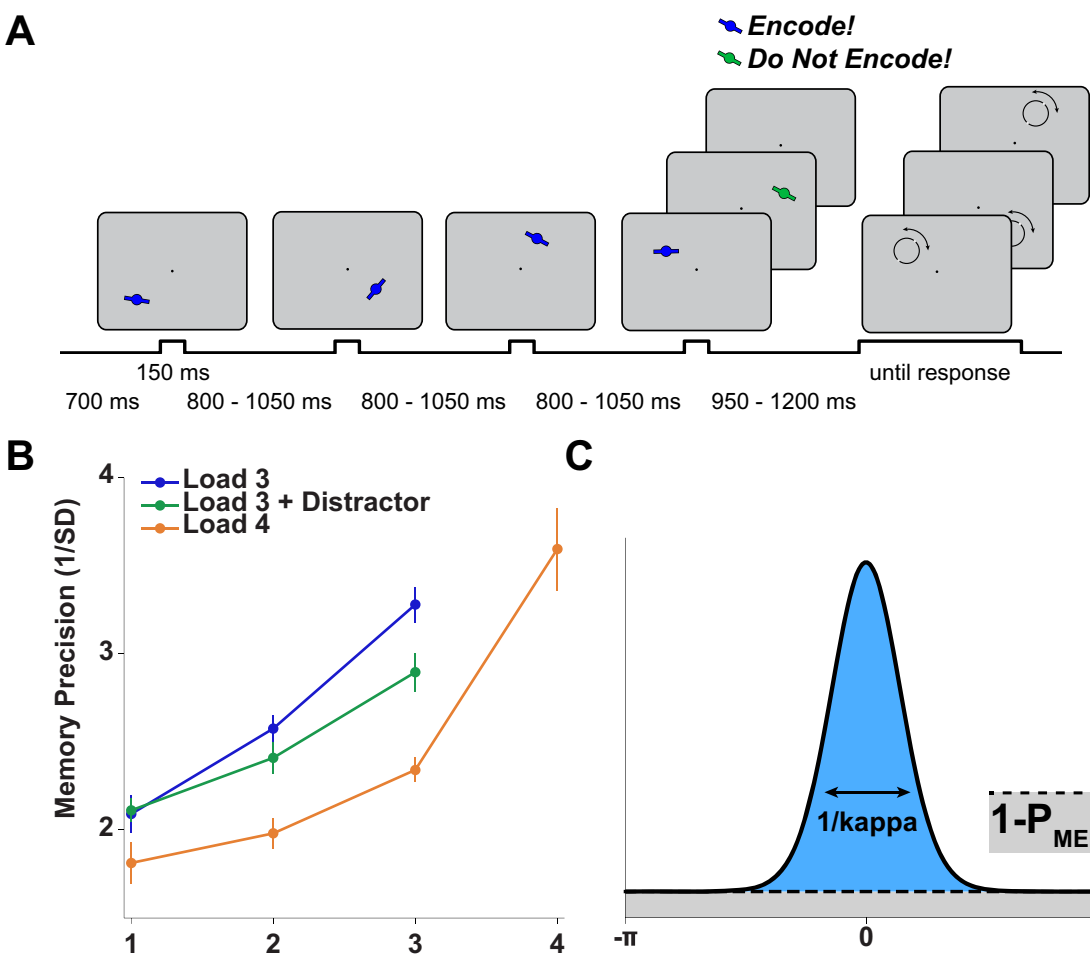

C

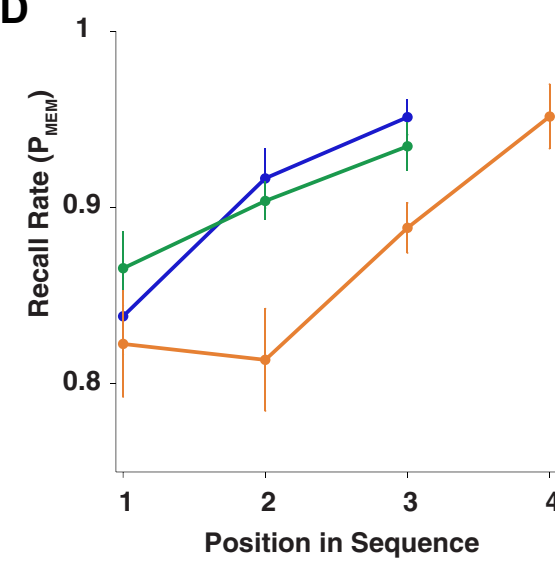

E

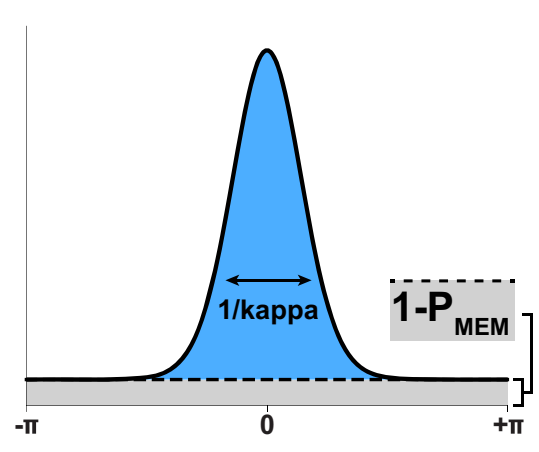

1. Design and behavioral results of the sequential WM task. $A$, Observers saw a temporally jittered sequence of at leas three oriented bars presented at different locations on the screen. 0n $70 \%$ of trials, a fourth bar appeared and could be either task relevant (blue, bottom row, probed in $25 \%$ of four-target trials) or task-irrelevant (green, middle row, never probed). Colors were counterbalanced across sessions. After a variable delay, a probe circle appeared at the location of one memory item. Observers indicated that item's orientation by moving indicator dots. After the response, the stimulus reappeared over the indicator dots as feedback (data not shown here). $\boldsymbol{B}$, The precision of the response distribution (i.e., the inverse of the circular SD) showed a robust recency effect for each condition. In addition, participants performed better on load 3 trials than on trials with a distractor or with a fourth relevant item. C, Schematic of response distribution consisting of a mixture of two underlying components: a uniform distribution driven by random guesses with height $1-P_{\text {MEM }}$ (gray shading) and a von Mises distribution driven by noisy recall with concentration $\kappa$ (blue shading).D, $\boldsymbol{E}$, Results of mixture-model fitting. We decomposed response distributions separately for each participant and condition, estimating the concentration $(\kappa)$ of the von Mises distribution and the recall rate $\left(P_{\text {MEM }}\right)$ for each. $\boldsymbol{D}$, Recall rates increased for items presented later in the trial and were higher on Load 3 and Load $3+D$ trials relative to Load 4 . $E$, Kappa also increased as a function of item position in the trial sequence and decreased as a function of load, with Load $3+D$ and Load 4 showing lower precision than Load 3. 
EEG acquisition. The EEG was recorded continuously using NuAmp amplifiers (Neuroscan) from $40 \mathrm{Ag} / \mathrm{AgCl}$ electrodes positioned according to the 10-20 international system (Sharbrough et al., 1991). Recordings were taken from electrodes as follows: $\mathrm{Fz}, \mathrm{FCz}, \mathrm{Cz}, \mathrm{CPz}, \mathrm{Pz}, \mathrm{POz}, \mathrm{Oz}$, FP1/2, F3/4, F7/8, FT7/8, T7/8, TP7/8, FC3/4, C3/4, CP3/4,P3/4, P7/8, $\mathrm{PO} 3 / 4, \mathrm{PO} 7 / 8$, and $\mathrm{O} 1 / 2$. Blinks and eye movements were monitored by deriving bipolar recordings from an electrode placed below the right eye and FP2 (VEOG) and from electrodes placed to the left and right of the right eye (HEOG).

The electrode placed at AFz served as the ground and the right mastoid served as the active reference. Electrical impedances were kept at $<5 \mathrm{k} \Omega$ and activity was filtered with a low-pass filter of $300 \mathrm{~Hz}$. The analog-todigital sampling rate of brain activity was set at $1000 \mathrm{~Hz}$ and data were recorded continuously for the entire experiment.

EEG processing. All further processing and analyses were done offline. First, the EEG was rereferenced to the average of the left and right mastoids. The two bipolar electrooculogram (EOG) signals were derived by calculating the difference between the upper and lower VEOG electrodes and between the left and right HEOG electrodes. Data were downsampled to $250 \mathrm{~Hz}$ and band-pass filtered between 0.10 and $80 \mathrm{~Hz}$. The filtered data were cut into trial-length epochs encompassing the stimulus presentation, memory delay, and probe presentation periods $(-1000$ to $5500 \mathrm{~ms}$ relative to trial onset). Slow linear, quadratic, and cubic drifts in the epochs were removed using a third-order polynomial fit performed separately for each channel and epoch. Control analyses on data that were neither filtered nor detrended yielded essentially the same results.

After rejecting epochs and channels with nonstereotyped artifacts (such as saturated channels), data were decomposed by independentcomponent analysis (ICA), as implemented in the EEGLAB toolbox (Delorme and Makeig, 2004), to identify components related to eye blinks and muscular artifacts, which were not included in the remixing of the data. After the ICA stage, trials with eye blinks (as identified in the EOG channels, which were not included in the ICA) and artifacts in a $\pm 100 \mathrm{~ms}$ window around any stimulus presentation were also discarded after visual inspection. The manual artifact removal step was added to remove trials in which eyeblinks may have precluded observers from seeing the stimulus (which is not addressed in the ICA step).

ERP analyses. To test for differences in the evoked responses to items as a function of task relevance (targets vs distractors), we calculated average evoked response potentials (ERPs) to each stimulus category. To this end, we temporally smoothed data with a Gaussian kernel (24 ms SD) and subtracted the average potential in the prestimulus baseline period (the $200 \mathrm{~ms}$ before a stimulus onset) before calculating subject-specific averages. We then used paired-samples $t$ tests (for the comparison between targets and distractors in the fourth position) to calculate grouplevel statistics. These results were corrected for multiple comparisons across time and sensors using 2D cluster-based permutation testing, with a cluster-forming threshold (and a cluster mass significance threshold) of $p=0.05$.

Correlation between alpha power and accuracy. To estimate trial-bytrial prestimulus power, we calculated power from 5 to $20 \mathrm{~Hz}$ (in $0.5 \mathrm{~Hz}$ steps) at each electrode using Morlet wavelets (with a length of 5 cycles) using MATLAB and the FieldTrip Analysis Toolbox (Oostenveld et al., 2011). We then calculated $10^{\star} \log _{10}$-transformed contralateral power (relative to the side of the stimulus, averaged across electrodes O1/2, $\mathrm{PO} 3 / 4 / 7 / 8$, and $\mathrm{P} 3 / 4 / 7 / 8$ ) at each time point around the onset time of the stimulus that would be probed on that trial $(-500$ to $+100 \mathrm{~ms})$.

For each frequency and each time point around the stimulus, we fit a general linear model (GLM) to the $z$-transformed contralateral alpha power using the $z$-transformed response accuracy as a regressor. Specifically, our measure of response accuracy was the negative absolute distance, in degrees, between the response angle and the tested stimulus angle, $-|x-\theta|$, where $x$ is the response angle and $\theta$ is the stimulus angle. Larger values (those closer to 0 ) therefore indicated more accurate performance. We accounted for the potentially confounding effects of sequence length ( 3 or 4 items), distractor presence, and sequence position of the tested item by fitting models for each of the 10 possible conditions separately and then averaging regression weights across conditions. To account for potential session effects on power (i.e., linear increases or decreases in power over the course of the experiment), we used trial number and session number as nuisance regressors in the GLM. Although trial number correlated positively with alpha power throughout the peristimulus interval, this had no effect on the correlation between contralateral alpha power and accuracy. Moreover, we saw no significant decrease in memory accuracy over the course of an experimental session, indicating that it had no functional impact.

At the group level, we used $t$ tests across the weights estimated in the GLM to test for a significant relationship between alpha lateralization and response accuracy at each frequency and each time point in the trial. We used cluster-based permutation testing to correct for multiple comparisons (across frequencies and time points, with 5000 permutations, a cluster-forming threshold of 0.05 , and a significance threshold of 0.05 ; Maris and Oostenveld, 2007).

To derive the topography of the correlation in the prestimulus period (500-100 ms before stimulus onset), we calculated trial-by-trial alpha power separately for each sensor. After averaging across frequencies (8-14 Hz in $0.5 \mathrm{~Hz}$ bins) and flipping topographies along the midline on trials where the stimulus was presented in the right hemifield (to visualize contralateral vs ipsilateral correlations), we performed the regression analysis separately at each sensor. We then used $t$ tests at each sensor and time point to arrive at group-level statistics.

Correlation between alpha phase and accuracy. We used the same timefrequency decomposition (Morlet wavelets from 5 to $20 \mathrm{~Hz}$ in $0.5 \mathrm{~Hz}$ steps) to estimate instantaneous phase at contralateral parietooccipital sensors (PO3/4/7/8, P3/4). We entered the circular average phase (across sensors) into a circular-linear correlation with memory accuracy (i.e., $-|x-\theta|)$, resulting in a time course of correlation values for each frequency. We then calculated the circular-linear correlation between alpha phase and 100 random permutations of memory accuracy (permuting across trials) and calculated the $z$-transformed rank (via the inverse normal cumulative distribution function) of the unpermuted correlation coefficient in the permutation distribution. We used one-sample $t$ tests to compare this $z$-score time course at the group level. Again, we used cluster-based permutation tests to calculate a corrected $p$-value. In addition, we applied the same approach at each sensor to calculate the topography of the peak of the phase-accuracy correlation in the prestimulus time window ( -350 to $-150 \mathrm{~ms}$ ). To compare the resulting topography with the topography of the alpha power effect, we used a standard topographical difference analysis (TANOVA; Murray et al., 2008). In brief, the difference between two topographies is the square root of the sum of squared differences at each sensor (after scaling each sensor by the variance across all sensors). This difference is compared with a permutation distribution to assess significance.

Modeling the effect of alpha power on precision and guess rate. Mathematical models of fluctuations in WM accuracy predict that fluctuations in alpha power should primarily affect the precision of memory, rather than the probability of recall (van den Berg et al., 2012). To test this hypothesis, we sought to correlate alpha power separately with trial-bytrial fluctuations in precision or in guess rate. To this end, we estimated (using maximum-likelihood estimation, MLE) the probability of recalling an item $\left(P_{\text {MEM }}\right)$ and the precision of remembered items $(\kappa)$ using the mixture model described in Equation 1.

After estimating these two parameters, we tested extended models that take trial-to-trial variations in alpha power into account. In the first model, we fit alpha power fluctuations to changes in precision as follows:

$$
\begin{array}{r}
p\left(x \mid \theta, \alpha_{P O W E R}\right)=\frac{P_{M E M}}{2 \pi I_{0}\left(\kappa\left(1+\beta^{\star} \alpha_{P O W E R}\right)\right)} e^{\kappa\left(1+\beta^{\star} \alpha_{P O W E R}\right) \cos (x-\theta)} \\
+\frac{1-P_{M E M}}{2 \pi} .
\end{array}
$$

where $\alpha_{\text {POWER }}$ is trial-by-trial and time point-by-time point posterior alpha power (averaged over $\mathrm{O} 1 / 2, \mathrm{PO} 3 / 4 / 7 / 8$, and $\mathrm{P} 3 / 4 / 7 / 8$ ) and $\beta$ is the influence of alpha power on precision (estimated using MLE).

In an alternative model, we fit alpha power to changes in recall rate as follows: 


$$
\begin{aligned}
p\left(x \mid \theta, \alpha_{P O W E R}\right)=\frac{P_{M E M}\left(1+\beta^{\star} \alpha_{P O W E R}\right)}{2 \pi I_{0}(\kappa)} e^{\kappa \cos (x-\theta)} & \\
& +\frac{1-P_{M E M}\left(1+\beta^{\star} \alpha_{P O W E R}\right)}{2 \pi} .
\end{aligned}
$$

The resulting parameter-estimate time courses were then tested at the group level using one-sample $t$ tests (under the null hypothesis that the average $\beta$ is 0 if alpha power has no effect). In addition, we fit this model to prestimulus alpha power at each sensor to obtain topographies of the effect.

We sought to validate this novel method using a more conservative approach. Here, we sorted prestimulus alpha power into two bins (separately for each behavioral condition) and fit the mixture model (see Eq. 1) separately to high-alpha-power and low-alpha-power trials. We then compared precision and recall rates between high and low alpha power using paired $t$ tests.

Link between visual evoked responses, prestimulus alpha power, and memory. If, as we argue, prestimulus alpha power is an index of cortical excitability, then it should predict the magnitude of visual responses to the memory stimulus. To test this hypothesis, we used the broadband EEG signal (band-pass filtered between 1 and $40 \mathrm{~Hz}$ and baseline corrected relative to 500 to $100 \mathrm{~ms}$ before stimulus onset, the time range of the prestimulus alpha power) as the dependent variable and correlated it with the trial-by-trial average of prestimulus contralateral posterior alpha power (using the same sensors and time window as above, i.e., 500$100 \mathrm{~ms}$ before stimulus onset). In addition, we used memory accuracy $(-|x-\theta|)$ as a second regressor (and trial number as a nuisance regressor). Our rationale for this analysis was the following: if alpha power modulates stimulus processing to improve performance, then both alpha power and accuracy should correlate with comparable epochs and sensors in the evoked signal.

To eliminate any possibly confounding effects of alpha power on prestimulus baseline potential, we conducted a control analysis in which we added each sensor's average prestimulus voltage as a nuisance regressor to the model. This did not noticeably affect the regression weights of alpha power and accuracy. To test the similarity of the correlation topographies with the ERP topography itself, we calculated the difference (TANOVA) between the mean ERP and the ERP-to-behavior and ERPto-alpha power correlations.

\section{Results}

\section{Behavioral results}

Observers completed a sequential working-memory precision task. After encoding three or four uniquely positioned peripheral items (see Materials and Methods for details) and maintaining them for a variable delay, a peripheral probe matching the location of one of the items prompted them to recall that item's orientation with a manual response. As expected, observers recalled more recently presented items with higher accuracy (main effect of item position in the trial sequence, $F_{(2,32)}=37.4, p<$ 0.001; see also Gorgoraptis et al., 2011). We also used a mixture model analysis to quantify separately the concentration of the Gaussian component $(\kappa)$ and recall rate $\left(P_{\mathrm{MEM}}\right.$, the average likelihood of recalling any information about a probed stimulus; see Materials and Methods and Fig. $1 D, E)$. More recently presented items were remembered with higher precision $\left(F_{(2,32)}=7.08, p=\right.$ $0.003)$ and with a higher recall rate $\left(F_{(2,32)}=7.89, p=0.002\right)$. Therefore, in the subsequent regression analyses, we calculated correlations between alpha oscillations and memory accuracy separately for each item position and then averaged regression weights across conditions. There was also a main effect of load on accuracy $\left(F_{(2,32)}=19.8, p<0.001\right)$, indicating that performance decreased when a distractor or a fourth target was presented. This effect was strongest when probing the third item in the sequence $\left(F_{(2,32)}=20.16, p<0.001\right)$, leading to a significant interaction between load and item position $\left(F_{(2,32)}=3.75, p<0.008\right)$. The mixture-model analysis showed that whereas the presentation of a fourth target decreased both the precision $\left(\mathrm{t}_{16}=4.45, p<\right.$ $0.001)$ and the recall rate for the third item $\left(t_{16}=3.76, p=\right.$ $0.002)$, presentation of a distractor only had a significant effect on precision $\left(\mathrm{t}_{16}=3.01, p=0.008\right)$, not on recall rate $\left(\mathrm{t}_{16}=1.37\right.$, $p=0.19)$.

\section{Prestimulus alpha power predicts precision of WM}

Spontaneous 8-14 Hz power before stimulus onset (at contralateral occipital-parietal sensors) predicted memory accuracy for the upcoming stimulus (two-tailed $t$ test, cluster-corrected $p=$ 0.008; Fig. $2 A$ ). The negative correlation indicates that memory was better when alpha power was lower; that is, when alpha was more desynchronized contralateral to the target. The correlation with accuracy was restricted to the alpha band and to the $500 \mathrm{~ms}$ preceding stimulus onset (Fig. 2A).

To evaluate the precise consequence of spontaneous alpha fluctuations on encoding, we used a model-based approach to test whether decreased alpha power predicted increases in the precision $(\kappa)$ or the recall rate $\left(P_{\mathrm{MEM}}\right)$ of WM. We found that alpha power fluctuations affected only the precision (cluster $p<$ 0.05 , again restricted to parietal and occipital sensors in the prestimulus epoch; Fig. $3 A, B$ ), but not the recall rate. A median split on prestimulus alpha power $(8-14 \mathrm{~Hz}, 500-100 \mathrm{~ms}$ before stimulus onset) confirmed significant differences in precision $\left(\mathrm{t}_{16}=\right.$ $2.15, p=0.024)$, but not in recall rate $\left(\mathrm{t}_{16}=-0.34, p>0.7\right.$, Fig. $3 C, D)$.

Further, we reasoned that, if higher excitability improves encoding, spontaneous decreases in excitability (as indexed by an increase in alpha power) might facilitate the inhibition of irrelevant distractors, thereby improving memory for previously encoded items. To this end, on a minority of trials (30\%), we presented a differently colored task-irrelevant distractor in the fourth position. On distractor trials, we correlated predistractor alpha power with accuracy for one of the preceding items. Initially, we used the same analysis approach as before targets; that is, correlating accuracy with contralateral power, and did not find a significant effect (cluster $p>0.25$ ). Given the relatively small number of trials for this analysis, we used the similar (but possibly more sensitive) approach of correlating the prestimulus lateralization (contralateral minus ipsilateral power). The alpha power lateralization before onset of the distractor predicted accuracy for the preceding items (cluster-corrected $p=0.023$; Fig. 4 , left). Here, we saw a positive correlation, indicating that alpha lateralization (higher power in contralateral relative to ipsilateral sensors) before a to-be-ignored distractor improves memory for the items already encoded into WM. We found no such effect for task-relevant unprobed items in the fourth position (Fig. 4, right). On average, the correlation before distractors (500-100 $\mathrm{ms}$ before stimulus onset) was higher than that before unprobed relevant items $\left(t_{16}=2.567, p=0.021\right)$. This result tentatively confirms that active inhibition at the point of encoding is required for this effect to occur.

\section{Prestimulus alpha phase correlates with behavior}

In addition, occipital-parietal alpha phase preceding the probed stimulus onset correlated significantly with memory performance (two-tailed $t$ test, cluster-corrected $p=0.035$; Fig. $2 B$ ). Again, the effect peaked $\sim 200 \mathrm{~ms}$ before stimulus onset. Similar to the alpha-power effects, the topography of alpha-phase effects was limited to posterior sensors (Fig. $2 B$ ), but was more broadly distributed. That said, we found no significant difference be- 

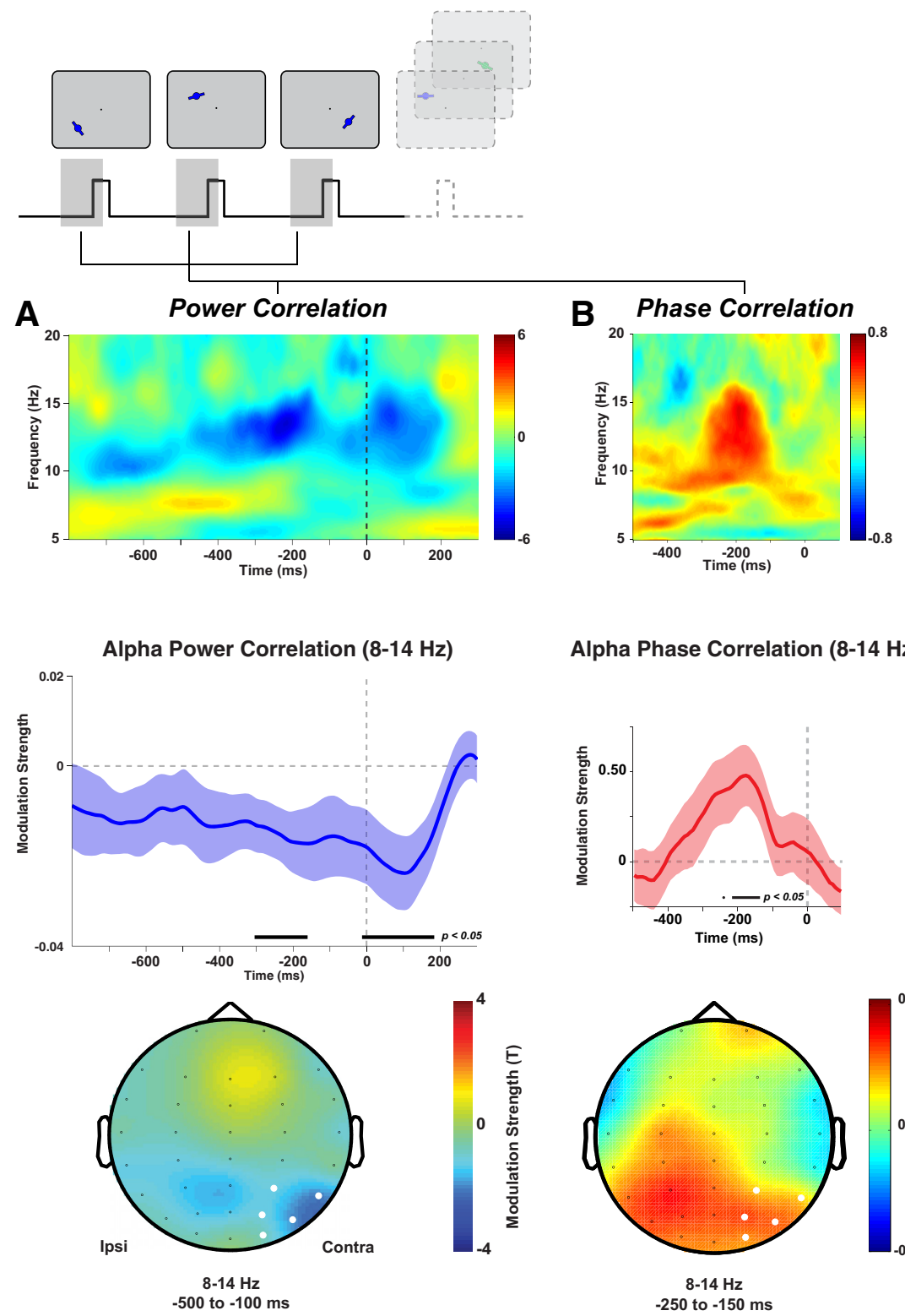

Alpha Phase Correlation (8-14 Hz)
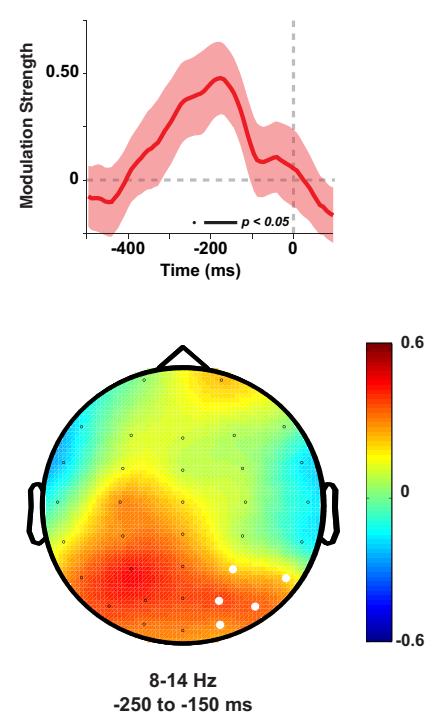

Figure 2. Posterior alpha oscillations predict WM accuracy. $A$, Lateralized posterior alpha power correlates with recall accuracy. Middle left, Alpha power lateralization before presentation of the probed stimulus correlates negatively with memory accuracy. Black bar indicates significant differences from 0 ( $t$ tests on regression weights from $\mathrm{GLM}, p<0.05$ ). Blue shading indicates SEM across participants. Top left, Time-frequency representation of the strength of correlation ( $t$ test on regression weights) showing a significant cluster in the alpha band preceding stimulus onset. Bottom left, Topographical distribution of regression weights preceding stimulus onset. White sensors were used for analyses in the two top panels. $\boldsymbol{B}$, Posterior alpha phase preceding stimulus onset predicts recall accuracy. The circular-linear correlation between the instantaneous alpha phase ( $8-14 \mathrm{~Hz}$, top right) on parietal sensors and response error increased $250-150$ ms before the onset of the probed stimulus (red line). Shading indicates SEM across participants. Black bar indicates a significant correlation ( $p<0.05$, permutation test). This prestimulus cluster is limited to the alpha band (top right). The plots show the z-scored circular-linear correlation coefficient (normalized relative to permutation distribution at each time point and frequency). Bottom right, Prestimulus increases in alpha phase correlations are restricted to parietal sensors (color indicates z-scored circular-linear correlation coefficient).

tween the average topographies $(500-100 \mathrm{~ms}$ prestimulus: $\mathrm{D}=$ $0.519, p=0.965 ; 250$ to $150 \mathrm{~ms}$ prestimulus: $\mathrm{D}=0.569, p=$ $0.935)$. Nevertheless, the apparently broader distribution compared with alpha power may result from alpha phase having a stronger spatial consistency across posterior sensors, leading to a spurious extension of the effect across the topography. To ensure that alpha phase was spontaneous and not entrained by the preceding stimulation, we calculated phase-locking values in the prestimulus period and found no significant phase locking in the alpha-band in the tested epochs (cluster $p>0.84$ ), confirming that the prestimulus alpha phase was randomly distributed. In contrast to the alpha power effect before the onset of a task-irrelevant distractor, we found no relationship between alpha phase and accuracy before distractor onset $(p>0.40$ on parietal-occipital electrodes).

It is possible that targets presented in the fourth position were processed differently from items presented earlier (because observers may have actively suppressed processing of the fourth item in anticipation of a possible distractor). When the trials probing the fourth item in the sequence were omitted from the analyses, we found essentially the same pattern of correlations between memory accuracy and contralateral alpha power (cluster $p=$ 0.018 ) and alpha phase (cluster $p=$ $0.061)$.

\section{Prestimulus alpha power and memory accuracy correlate with parietal evoked potential amplitude}

In a final analysis, we examined how visual evoked responses might form a mechanistic link between changes in cortical state (i.e., alpha power) and memory. We used multiple regression to correlate the trial-by-trial stimulus-evoked EEG with prestimulus alpha power and memory accuracy. Both alpha power and accuracy showed a peak in correlation over posterior sensors $\sim 200 \mathrm{~ms}$ after stimulus onset (Fig. 5, middle). This peak corresponds in time with the lateral posterior N1 potential (Fig. 5, top), but is strongest over central parietal sensors (for the correlation with accuracy). Consistent with this topographical discrepancy, we found that the ERP topography (200-250 ms after stimulus) differed from the topographies of the ERP-accuracy correlation $(\mathrm{D}=0.725, p=0.053)$ and the ERP-alpha power correlation $(\mathrm{D}=0.910, p=0.004)$. Effects of alpha power and accuracy, therefore, may have involved only a subset of the sources active in this time window. The correlation with alpha power was positive, indicating that decreases in alpha power increased the amplitude of the (negative-going) ERP. Conversely, the correlation with accuracy was negative, indicating that, for highaccuracy trials, the ERP was more negative. Both correlations peaked over parietooccipital sensors (Fig. 5, bottom), although the peak of the accuracy correlation was more central, whereas alpha power also correlated with posterolateral sensors.

\section{Broadband effects of distractor processing}

Because prestimulus alpha power preceding a distractor predicted memory accuracy for previous items whereas alpha pre- 
A

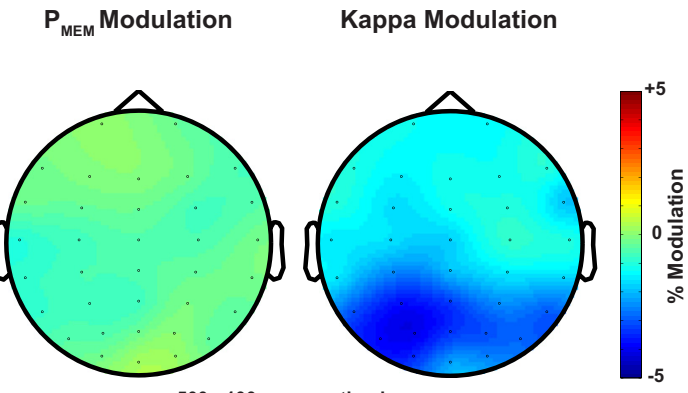

$500-100$ ms pre-stimulus

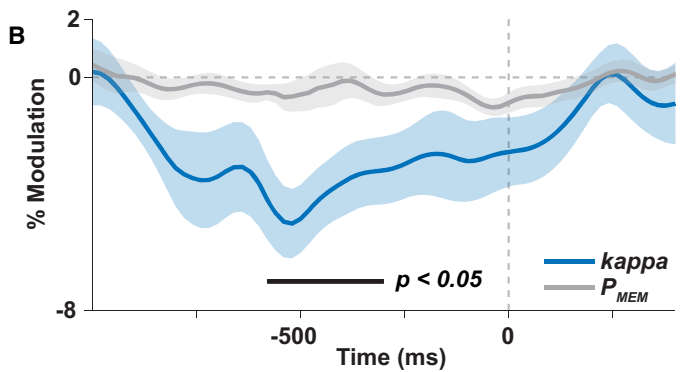

C
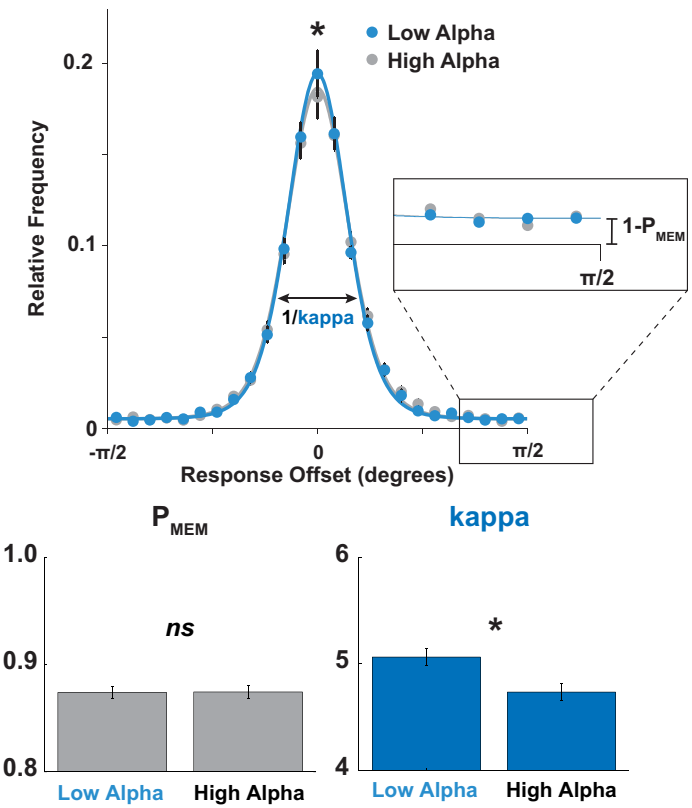

Figure 3. Prestimulus alpha power predicts changes in memory precision, but not in guess rate. Using a model-based analysis, we estimated the parametric influence of alpha power on precision and guess rate in the memory mixture model. Although there was no significant effect on guess rate, we found significant effects (cluster-corrected $p<0.05$ ) of alpha power on precision. This effect peaked over posterior sensors $(\boldsymbol{A}) \sim 250$ to $500 \mathrm{~ms}$ before stimulus onset ( $\boldsymbol{B}$, significant differences between precision modulation and guess rate modulation indicated by the black bar). $\boldsymbol{C}$, Histogram of responses, split into high- and low-alpha trials (binned separately for each condition and then merged). The only significant difference between high-and low-alpha trials was found in the height of the histogram peak, with low alpha trials producing more high-precision responses $(p<0.05)$. $\boldsymbol{D}$, This effect was reflected in a significant difference between precision on high- and low-alpha trials $(p=0.024)$, Guess rate did not differ between high- and low-alpha trials $(p>0.70)$.

ceding a relevant but unprobed fourth target item did not, we wanted to ensure that these two types of stimuli were indeed processed differently by the visual system (see Materials and Methods). We found early frontal differences between targets and distractors (cluster-corrected $p<$ 0.05 for two clusters) that were followed closely by a relatively early dissociation in the posterior visual evoked response (Fig. 6 ), with significant differences in the topographies after $200 \mathrm{~ms}$. These differences may account for the different behavioral effects of targets and distractors (Fig. 1B).

\section{Discussion}

Our results indicate that spontaneous changes in visual excitability, measured by oscillations in the alpha band, contribute to the representational precision of WM encoding. We found this effect even though stimuli were presented in isolation and well above the contrast threshold, suggesting that alpha-band synchronization can bias encoding even when stimuli are easily detectable and are not competing directly for attention.

Using a model-based analysis approach, we found evidence that posterior alpha power predominantly influences the precision of memory for an item, rather than the likelihood that it will be robustly encoded in WM. This finding fits
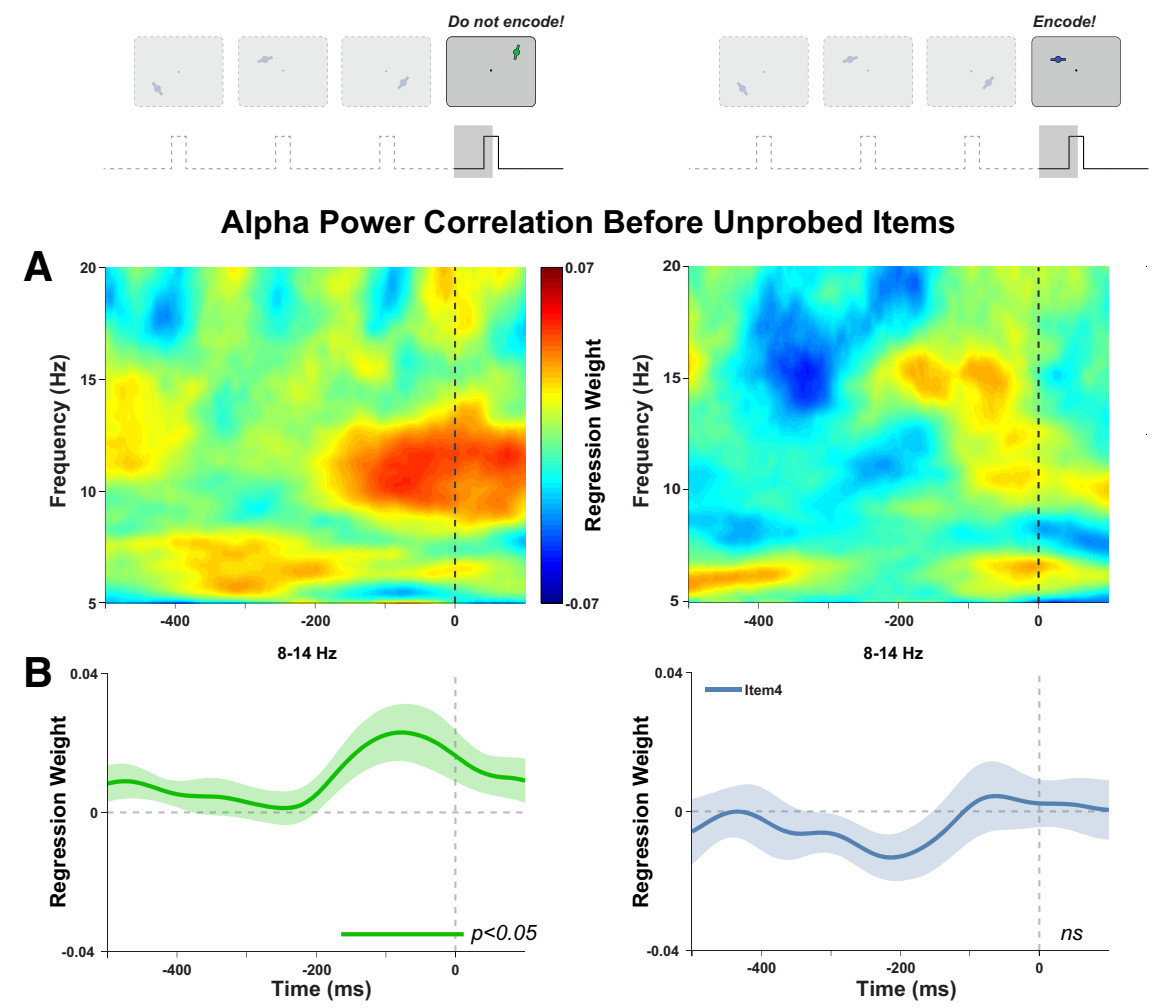

Figure 4. Alpha power lateralization before an irrelevant distractor predicts memory accuracy for preceding items. A, Posterior alpha lateralization before onset of a distractor at position 4 positively correlates with response errors to items 1,2 , or 3 . This effect was again restricted to the alpha range (cluster corrected $p=0.02$ ). We saw no correlation with response errors to items 1,2 , or 3 when a task-relevant item was presented at position 4 (cluster $p>0.20$, right). Note that this is distinct from the correlation before the probed item shown in Figure 2. B, Significant correlations with accuracy in the alpha band $(8-14 \mathrm{~Hz})$ began $\sim 200 \mathrm{~ms}$ before stimulus onset. 

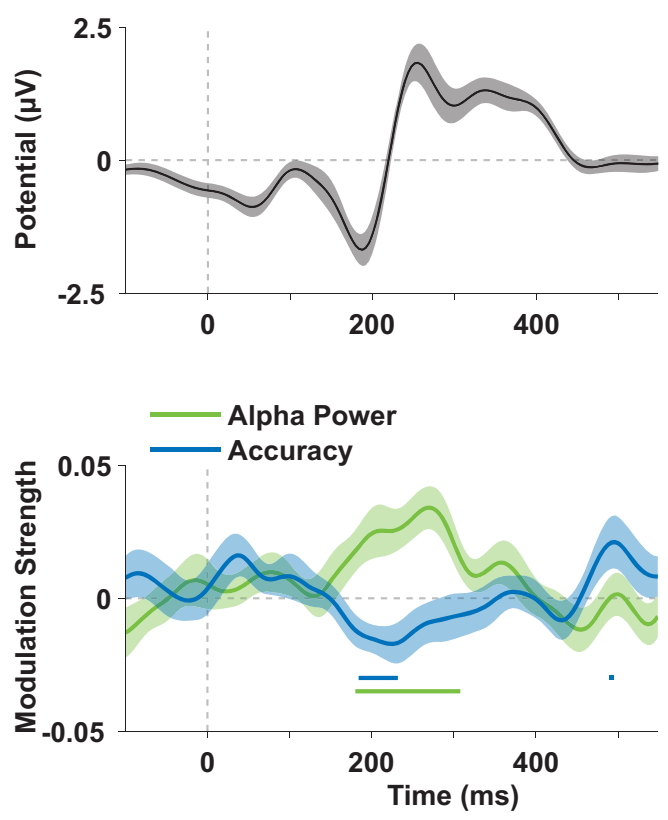

VSTM Accuracy
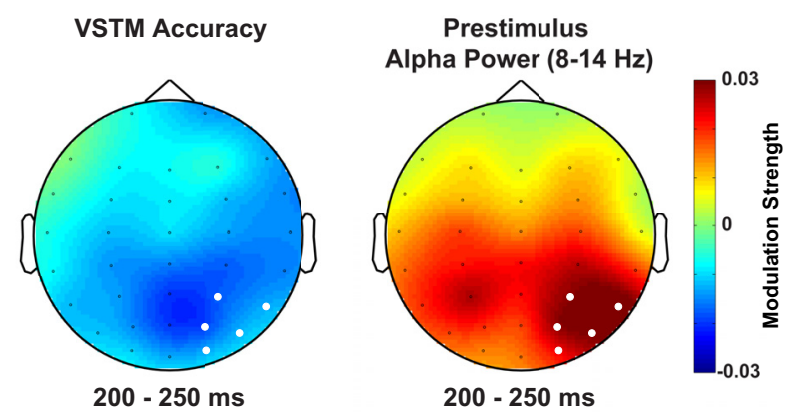

Figure 5. Prestimulus alpha power and memory accuracy correlate with posterior visual ERPs. Potentials on sensors contralateral to the stimulus (highlighted in white on the topographies, bottom) correlated significantly with posterior contralateral 8-14 Hz power (calculated at the same sensors, 500 to 100 ms before stimulus onset) and with memory accuracy ( $-\mid x-$ $\theta$ |) 200 to $250 \mathrm{~ms}$ after stimulus onset (see regression time courses, middle). The time range (shaded blue and green boxes, top) when both variables correlated significantly with broadband EEG corresponds approximately to the time of the negative-going N1 potential. This indicates that higher alpha power, which showed a positive correlation (green line, middle), tended to decrease the (negative) amplitude of the EEG, whereas accuracy (blue line, middle) was higher when the ERP was more negative (as indicated by a negative correlation). Both correlations peaked over posterior parietal and occipital sensors (bottom). Shaded errors indicate SEM.

predictions of recent mathematical models of WM, which have argued that stochastic variability in neural gain influences the neural precision of encoded information (Ma et al., 2006; van den Berg et al., 2012). One alternative model (Sims et al., 2012) proposed that the variability in WM can be explained by variability in how many items are encoded from a multiitem display (while encoding precision is constant). Research into the separate neural contributions to limits in quality and quantity of WM is only beginning to emerge (Anderson et al., 2011; Machizawa et al., 2012; Emrich et al., 2013). Although our study favors the interpretation that spontaneous alpha fluctuations influence precision, it does not contradict models emphasizing variability in the quantity of encoded items. Other state variables, such as excitability in prefrontal cortex or connectivity between prefrontal and visual areas (Curtis and D'Esposito, 2003; Gazzaley et al., 2004; Zanto et al., 2011), could influence how many items are encoded into WM on a trial-to-trial basis. Similarly, an influence
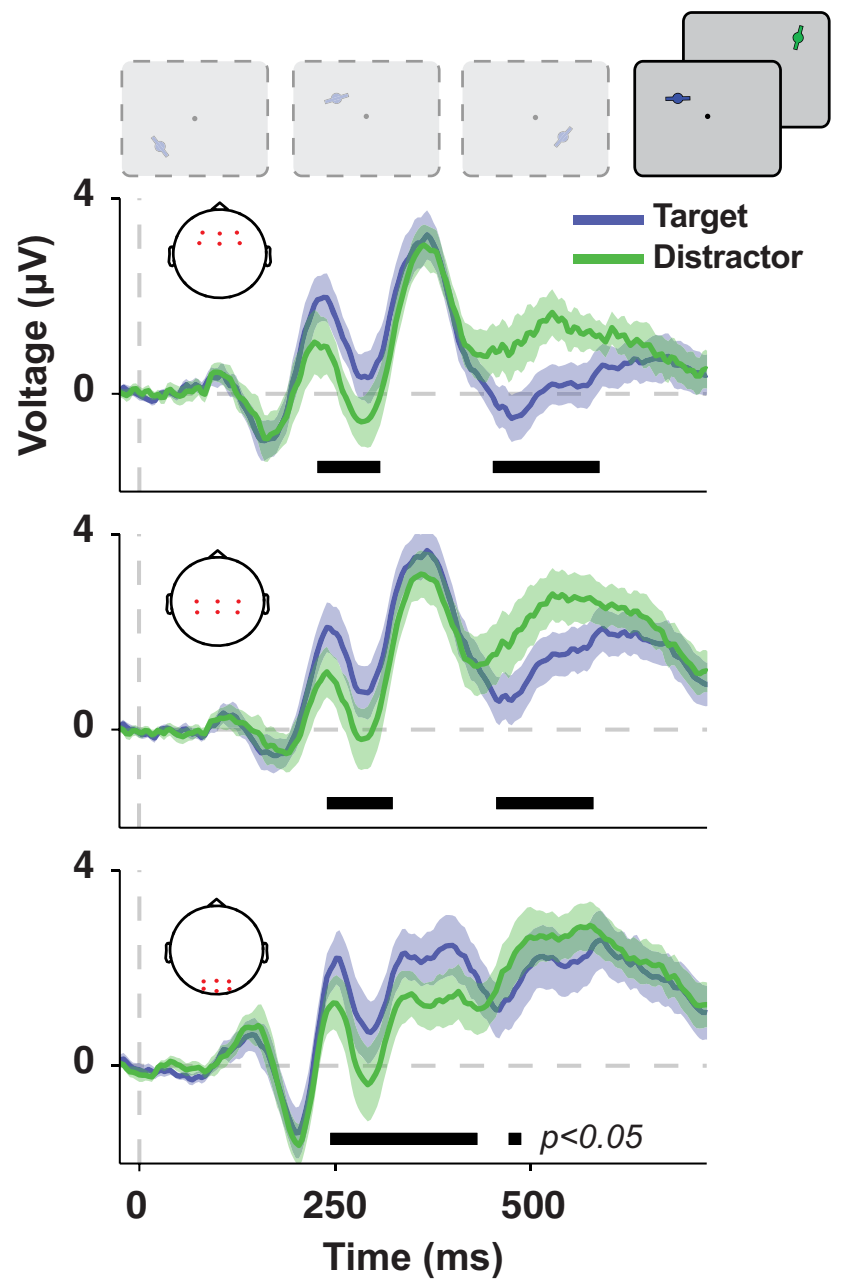

Figure 6. Broadband responses to task-relevant and irrelevant stimulus onsets. Shown here are average ERPs for frontal, central, and posterior electrodes (electrode locations shown in insets) separately for targets presented in position 4 (blue) and for distractors in position 4 (green). We compared targets with distractors and found significant differences in three clusters $(p<0.05)$. Targets led to an enhanced P2 ( $200-275 \mathrm{~ms}$ ) and P3 ( $300-425 \mathrm{~ms}$ ) response and an attenuation of the sustained response at $\sim 500 \mathrm{~ms}(450-600 \mathrm{~ms})$. Shaded areas around the ERP time courses represent within-subject SEM. Black bars indicate time points when target and distractor-evoked potentials are significantly different ( $p<0.05$, uncorrected).

on quantity might depend on perceptual competition between items. Here, we explicitly avoided low-level interitem competition by presenting stimuli in sequence. This design allowed us to show that the prestimulus state has long-lasting consequences for memory even when perceptual constraints are minimized. It is possible that prestimulus alpha power could also influence the probability of encoding specific items in a design using simultaneous presentation of multiple items.

Before a distractor, we found evidence that lateralization of alpha power in the opposite direction (i.e., a relative increase in contralateral, compared with ipsilateral, alpha power) improves memory for a previously presented item. We found no such effect before an unprobed task-relevant item, possibly indicating that the correlation is specific to items that are actively inhibited during visual processing (as may be borne out in processing differences between targets and distractors; Fig. 6). However, because the difference between distractors and target items was modest, future studies could use a more powerful design to test for differential effects preceding task-relevant or irrelevant items. This result complements a recent finding that alpha power before an 
expected, centrally presented distractor predicts the speed of recall from WM (Bonnefond and Jensen, 2012). In that study, distractors appeared on every trial with predictable timings and distractor strength was manipulated between blocks. The predictability of the distractor item presumably allowed for precise top-down control over alpha synchronization by temporal expectations (Rohenkohl and Nobre, 2011). Moreover, because distractors were centrally presented, the effects on alpha were distributed across central posterior sensors. In contrast, in the present study, overall alpha power did not significantly predict memory performance, whereas the more spatially specific lateralization did significantly covary with accuracy of the taskrelevant items. Although our results are broadly consistent with Bonnefond and Jensen (2012), differences in the predictability of the distractor item presumably account for differences in the precise pattern of prestimulus effects.

For task-relevant items that were probed, we also found a relationship between prestimulus alpha phase and WM performance. This finding may seem counterintuitive at first glance: because the presentation time of each stimulus (150 ms) was longer than one alpha cycle ( $\sim 100 \mathrm{~ms})$, our design should have allowed sufficient opportunity for stimulus processing to occur at the optimal alpha phase. However, the alpha phase preceding stimulus onset may affect the number of alpha duty cycles occurring during stimulus presentation. More phases of high excitability should therefore affect the quality of encoding (for a similar argument, see Hanslmayr et al., 2013). Alternatively (or additionally), in a dynamical system such as the brain, small shifts in initial conditions (caused by variability in alpha phase) may have longlasting effects on the neural trajectory of stimulus processing. Because stimulus onsets were temporally jittered, observers were unable to predict the exact onset time of the next target. It is therefore unlikely that they were able to use anticipatory topdown control to entrain alpha oscillations to a behaviorally optimal phase. This strengthens our conclusion that stochastic fluctuations in alpha (phase and power) were directly responsible for WM improvements. The lack of strategic control over alpha phase may also explain why phase before a distractor had no relationship with accuracy, which is in contrast to Bonnefond and Jensen, (2012), in which the temporal predictability of the distractor onset was thought to have played an important role.

We suggest that amplification of visual evoked potentials may be a mechanism by which prestimulus alpha influences memory. In accordance with previous studies (Brandt and Jansen, 1991; Brandt et al., 1991), we found correlations between prestimulus alpha power and the amplitude of evoked responses $\sim 200 \mathrm{~ms}$ after stimulus onset (for effects on earlier components, see Rajagovindan and Ding, 2011). Around the same time, ERP amplitude also correlated with memory accuracy, indicating that the modulation of visual ERPs could mediate between prestimulus state and ultimate memory performance. It should be noted that the topography of the correlation with accuracy had a more central-parietal peak than the posterolateral correlations with alpha power. Although the coarse spatial resolution of EEG precludes any strong conclusions from this shift, it does point to the possibility that the link between alpha fluctuations and behavior may depend on facilitating a cascade of multiple processing stages occurring in different areas and at different time points during encoding. An intriguing possibility is that this initial state influences the latency of certain processing steps during encoding, leading to a more efficient trajectory through neural state space (Harvey et al., 2012) to reach a stable memory state (Stokes et al., 2013). The correlation between memory accuracy and the visual
ERP spanned both the peak of the N1 and the onset of the P2, so that we are not able to disambiguate the exact point of influence. Subsequent studies are needed to identify the specific influence of the prestimulus state on the processing cascade of WM encoding.

The present study differs from many related experiments in that it examined the effect of spontaneous electrophysiological dynamics on behavior, rather than the effect of top-down attentional cues on behavior and electrophysiology. Such cues can have enduring consequences for WM (Gazzaley and Nobre, 2012; Nobre and Stokes, 2011). Attention during encoding improves memory (Rutman et al., 2010; Gazzaley, 2011) and has been linked to electrophysiological markers of endogenous attention shifts (Murray et al., 2011). Possibly, top-down attention constrains the variability in firing rate (Churchland et al., 2010; Cohen and Maunsell, 2010; Churchland et al., 2011), leading to increased encoding precision. Future studies could test this proposal through a direct comparison of spontaneous and cueevoked variability in the alpha state and how this relates to stimulus-evoked response variability. The ability to control spontaneous fluctuations in neural excitability could be linked to interindividual variability in WM capacity (Vogel and Machizawa, 2004; Vogel et al., 2005; Sauseng et al., 2009). Finally, single-unit electrophysiology or magnetoencephalography studies will need to test whether alpha oscillations indeed act on WM by increasing firing rates or high-frequency (gamma-band) evoked power in visual cortex, allowing us to formulate a stronger mechanistic link between spontaneous state changes and task performance.

\section{References}

Anderson DE, Vogel EK, Awh E (2011) Precision in visual working memory reaches a stable plateau when individual item limits are exceeded. J Neurosci 31:1128-1138. CrossRef Medline

Bays PM, Catalao RF, Husain M (2009) The precision of visual working memory is set by allocation of a shared resource. J Vis 9:7.1-11. CrossRef Medline

Bollimunta A, Mo J, Schroeder CE, Ding M (2011) Neuronal mechanisms and attentional modulation of corticothalamic alpha oscillations. J Neurosci 31:4935-4943. CrossRef Medline

Bonnefond M, Jensen O (2012) Alpha oscillations serve to protect working memory maintenance against anticipated distracters. Curr Biol 22:19691974. CrossRef Medline

Brandt ME, Ben H Jansen (1991) The relationship between prestimulus alpha amplitude and visual evoked potential amplitude. international J Neurosci 61:261-268. CrossRef

Brandt ME, Jansen BH, Carbonari JP (1991) Pre-stimulus spectral EEG patterns and the visual evoked response. Electroencephalogr Clin Neurophysiol 80:16-20. CrossRef Medline

Busch NA, VanRullen R (2010) Spontaneous EEG oscillations reveal periodic sampling of visual attention. Proc Natl Acad Sci U S A 107:1604816053. CrossRef Medline

Chakravarthi R, Vanrullen R (2012) Conscious updating is a rhythmic process. Proc Natl Acad Sci U S A 109:10599-10604. CrossRef Medline

Churchland AK, Kiani R, Chaudhuri R, Wang XJ, Pouget A, Shadlen MN (2011) Variance as a signature of neural computations during decision making. Neuron 69:818-831. CrossRef Medline

Churchland MM, Yu BM, Cunningham JP, Sugrue LP, Cohen MR, Corrado GS, Newsome WT, Clark AM, Hosseini P, Scott BB, Bradley DC, Smith MA, Kohn A, Movshon JA, Armstrong KM, Moore T, Chang SW, Snyder LH, Lisberger SG, Priebe NJ, et al. (2010) Stimulus onset quenches neural variability: a widespread cortical phenomenon. Nat Neurosci 13:369378. CrossRef Medline

Cohen MR, Maunsell JH (2010) A neuronal population measure of attention predicts behavioral performance on individual trials. J Neurosci 30: 15241-15253. CrossRef Medline

Cowan N (2000) The magical number 4 in short-term memory: a reconsideration of mental storage capacity. Behav Brain Sci 24:87-114; discussion 114-185. Medline

Curtis CE, D’Esposito M (2003) Persistent activity in the prefrontal cortex 
during working memory. Trends in Cognitive Sciences 7:415-423. CrossRef Medline

Delorme A, Makeig S (2004) EEGLAB: an open source toolbox for analysis of single-trial EEG dynamics including independent component analysis. J Neurosci Methods 134:9-21. CrossRef Medline

Emrich SM, Riggall AC, Larocque JJ, Postle BR (2013) Distributed patterns of activity in sensory cortex reflect the precision of multiple items maintained in visual short-term memory. J Neurosci 33:6516-6523. CrossRef Medline

Engle RW, Tuholski SW, Laughlin JE, Conway AR (1999) Working memory, short-term memory, and general fluid intelligence: A latent-variable approach. J Exp Psychol Gen 128:309-331. CrossRef Medline

Fougnie D, Suchow JW, Alvarez GA (2012) Variability in the quality of visual working memory. Nat Commun 3:1229. CrossRef Medline

Fukuda K, Vogel E, Mayr U, Awh E (2010) Quantity, not quality: the relationship between fluid intelligence and working memory capacity. Psychon Bull Rev 17:673-679. CrossRef Medline

Gathercole SE, Pickering SJ, Knight C, Stegmann Z (2003) Working memory skills and educational attainment: evidence from national curriculum assessments at 7 and 14 years of age. Applied Cognitive Psychology 18:1-16.

Gazzaley A (2011) Influence of early attentional modulation on working memory. Neuropsychologia 49:1410-1424. CrossRef Medline

Gazzaley A, Nobre AC (2012) Top-down modulation: bridging selective attention and working memory. Trends Cogn Sci 16:129-135. CrossRef Medline

Gazzaley A, Rissman J, D'Esposito M (2004) Functional connectivity during working memory maintenance. Cogn Affect Behav Neurosci 4:580-599. CrossRef Medline

Gorgoraptis N, Catalao RF, Bays PM, Husain M (2011) Dynamic updating of working memory resources for visual objects. J Neurosci 31:85028511. CrossRef Medline

Gould IC, Rushworth MF, Nobre AC (2011) Indexing the graded allocation of visuospatial attention using anticipatory alpha oscillations. J Neurophysiol 105:1318-1326. CrossRef Medline

Haegens S, Nácher V, Luna R, Romo R, Jensen O (2011) $\alpha$-Oscillations in the monkey sensorimotor network influence discrimination performance by rhythmical inhibition of neuronal spiking. Proc Natl Acad Sci U S A 108:19377-19382. CrossRef Medline

Hanslmayr S, Aslan A, Staudigl T, Klimesch W, Herrmann CS, Bäuml KH (2007) Prestimulus oscillations predict visual perception performance between and within subjects. Neuroimage 37:1465-1473. CrossRef Medline

Hanslmayr S, Volberg G, Wimber M, Dalal SS, Greenlee MW (2013) Prestimulus oscillatory phase at $7 \mathrm{~Hz}$ gates cortical information flow and visual perception. Curr Biol 23:2273-2278. CrossRef Medline

Harvey CD, Coen P, Tank DW (2012) Choice-specific sequences in parietal cortex during a virtual-navigation decision task. Nature 484:62-68. CrossRef Medline

Jensen O, Mazaheri A (2010) Shaping functional architecture by oscillatory alpha activity: gating by inhibition. Front Hum Neurosci 4:186. CrossRef Medline

Keshvari S, van den Berg R, Ma WJ (2013) No evidence for an item limit in change detection. PLoS Comput Biol 9:e1002927. CrossRef Medline

Klimesch W, Sauseng P, Hanslmayr S (2007) EEG alpha oscillations: the inhibition-timing hypothesis. Brain Res Rev 53:63-88. CrossRef Medline

Luck SJ, Vogel EK (2013) Visual working memory capacity: from psychophysics and neurobiology to individual differences. Trends Cogn Sci 17: 391-400. CrossRef Medline

Ma WJ, Beck JM, Latham PE, Pouget A (2006) Bayesian inference with probabilistic population codes. Nat Neurosci 9:1432-1438. CrossRef Medline

Machizawa MG, Goh CC, Driver J (2012) Human visual short-term memory precision can be varied at will when the number of retained items is low. Psychol Sci 23:554-559. CrossRef Medline

Maris E, Oostenveld R (2007) Nonparametric statistical testing of EEG-and MEG-data. J Neurosci Methods 164:177-190. CrossRef Medline

Mathewson KE, Lleras A, Beck DM, Fabiani M, Ro T, Gratton G (2011) Pulsed out of awareness: EEG alpha oscillations represent a pulsed- inhibition of ongoing cortical processing. Front Psychol 2:99. CrossRef Medline

Murray AM, Nobre AC, Stokes MG (2011) Markers of preparatory attention predict visual short-term memory performance. Neuropsychologia 49:1458-1465. CrossRef Medline

Murray MM, Brunet D, Michel CM (2008) Topographic ERP analyses: a step-by-step tutorial review. Brain Topogr 20:249-264. CrossRef Medline

Nobre AC, Stokes MG (2011) Attention and short-term memory: crossroads. Neuropsychologia 49:1391-1392. CrossRef Medline

Oostenveld R, Fries P, Maris E, Schoffelen JM (2011) FieldTrip: open source software for advanced analysis of MEG, EEG, and invasive electrophysiological data. Comput Intell Neurosci 2011:156869. CrossRef Medline

Orhan AE, Jacobs RA (2013) A probabilistic clustering theory of the organization of visual short-term memory. Psychol Rev 120:297-328. CrossRef Medline

Rajagovindan R, Ding M (2011) From prestimulus alpha oscillation to visual-evoked response: an inverted-U function and its attentional modulation. J Cogn Neurosci 23:1379-1394. CrossRef Medline

Rohenkohl G, Nobre AC (2011) Alpha oscillations related to anticipatory attention follow temporal expectations. J Neurosci 31:14076-14084. CrossRef Medline

Romei V, Rihs T, Brodbeck V, Thut G (2008) Resting electroencephalogram alpha-power over posterior sites indexes baseline visual cortex excitability. Neuroreport 19:203-208. CrossRef Medline

Rutman AM, Clapp WC, Chadick JZ, Gazzaley A (2010) Early top-down control of visual processing predicts working memory performance. J Cogn Neurosci 22:1224-1234. CrossRef Medline

Saalmann YB, Pinsk MA, Wang L, Li X, Kastner S (2012) The pulvinar regulates information transmission between cortical areas based on attention demands. Science 337:753-756. CrossRef Medline

Sauseng P, Klimesch W, Heise KF, Gruber WR, Holz E, Karim AA, Glennon M, Gerloff C, Birbaumer N, Hummel FC (2009) Brain oscillatory substrates of visual short-term memory capacity. Curr Biol 19:1846-1852. CrossRef Medline

Sharbrough F, Chatrian GE, Lesser RP, Lüders H (1991) American Electroencephalographic Society guidelines for standard electrode position nomenclature. J Clin Neurophysiol.

Sims CR, Jacobs RA, Knill DC (2012) An ideal observer analysis of visual working memory. Psychol Rev 119:807-830. CrossRef Medline

Spaak E, Bonnefond M, Maier A, Leopold DA, Jensen O (2012) Layerspecific entrainment of gamma-band neural activity by the alpha rhythm in monkey visual cortex. Curr Biol 22:2313-2318. CrossRef Medline

Stokes MG, Kusunoki M, Sigala N, Nili H, Gaffan D, Duncan J (2013) Dynamic coding for cognitive control in prefrontal cortex. Neuron 78:364375. CrossRef Medline

van den Berg R, Shin H, Chou WC, George R, Ma WJ (2012) Variability in encoding precision accounts for visual short-term memory limitations. Proc Natl Acad Sci U S A 109:8780-8785. CrossRef Medline

van Dijk H, Schoffelen JM, Oostenveld R, Jensen O (2008) Prestimulus oscillatory activity in the alpha band predicts visual discrimination ability. J Neurosci 28:1816-1823. CrossRef Medline

VanRullen R, Macdonald JS (2012) Perceptual echoes at $10 \mathrm{~Hz}$ in the human brain. Curr Biol 22:995-999. CrossRef Medline

Vogel EK, Machizawa MG (2004) Neural activity predicts individual differences in visual working memory capacity. Nature 428:748-751. CrossRef Medline

Vogel EK, McCollough AW, Machizawa MG (2005) Neural measures reveal individual differences in controlling access to working memory. Nature 438:500-503. CrossRef Medline

Wyart V, Sergent C (2009) The phase of ongoing EEG oscillations uncovers the fine temporal structure of conscious perception. J Neurosci 29: 12839-12841. CrossRef Medline

Zanto TP, Rubens MT, Thangavel A, Gazzaley A (2011) Causal role of the prefrontal cortex in top-down modulation of visual processing and working memory. Nat Neurosci 14:656-661. CrossRef Medline

Zhang W, Luck SJ (2008) Discrete fixed-resolution representations in visual working memory. Nature 453:233-235. CrossRef Medline 